\title{
Neutrophil chemotaxis in granulomatosis with polyangiitis (Wegener's) and idiopathic pulmonary fibrosis
}

\author{
A.G. Richter*, G.D. Perkins”, A. Chavda*, E. Sapey*, L. Harper ${ }^{\star}$ and D.R. Thickett*
}

ABSTRACT: The presence of antineutrophil cytoplasmic antibodies in granulomatosis with polyangiitis (Wegener's) (GPA) implicates the neutrophil as a key effector cell. Previous studies have reported elevated neutrophil counts in the lung, although the determinants of neutrophil chemotaxis in the GPA lung are unknown.

Bronchoalveolar lavage fluid (BALF) cell counts, myeloperoxidase (MPO) and chemokines were measured in 27 patients with GPA, 20 disease controls with idiopathic pulmonary fibrosis (IPF) and six healthy controls. CXC chemokine ligand (CXCL)8, interleukin (IL)-1ß, epithelial neutrophilactivating protein 78, granulocyte colony-stimulating factor (G-CSF) and granulocyte-macrophage colony-stimulating factor were measured by ELISA. The neutrophil chemotactic potential of BALF was investigated using the under-agarose method, and specific antibodies were used to examine the role of CXCL8 and IL-1ק.

GPA BALF had an increased neutrophil percentage, and elevated MPO, CXCL8 and G-CSF concentrations compared with healthy controls. Chemotaxis of control neutrophils towards BALF from patients with active $(p=0.006)$ and remission $(p=0.077)$ GPA, and IPF $(p=0.001)$ patients was increased compared with normal controls. BALF-induced chemotaxis correlated with BALF IL-1 $\beta$ $(r=0.761, p=0.001)$ and CXCL8 $(r=0.640, p=0.012)$ in GPA, and was inhibited by anti-CXCL8 $(85 \%$; $p<0.001)$ and anti-IL-1 $\beta(69 \% ; p<0.001)$.

Our study confirms a neutrophilia and pro-inflammatory alveolar milieu that persists in clinical remission. CXCL8 and IL-1 $\beta$ appear to play important roles in the neutrophil chemotactic response to BALF.

KEYWORDS: Bronchoalveolar lavage, chemokines, interstitial lung disease, neutrophil, Wegener's granulomatosis

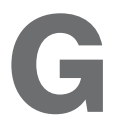
ranulomatosis with polyangiitis (Wegener's) (GPA) is a small-vessel, systemic vasculitis, which is characterised immunologically by antineutrophil cytoplasmic antibodies (ANCAs), predominantly directed against the neutrophil serine proteinase (PR)-3, but also myeloperoxidase (MPO) [1]. The presence of ANCAs implicates the neutrophil as a key effector cell in the pathogenesis of GPA. In vitro, ANCAs activate primed neutrophils, resulting in an enhanced respiratory burst and cytokine release [2]. An animal model of ANCA-associated vasculitis suggested that neutrophils play a direct role in the induction of disease [3] and an in vivo study revealed that activated neutrophils within glomeruli correlated with the severity of renal injury [4].

GPA frequently involves the lungs [5, 6], with one series suggesting up to $90 \%$ of patients suffer from lower respiratory tract involvement at some stage during the disease course [7]. Increased neutrophil levels have been previously reported in the bronchoalveolar lavage fluid (BALF) from GPA patients during active disease $[8,9]$. Unchecked neutrophilic inflammation contributes to the tissue damage seen in other chronic lung disorders, such as bronchiectasis [10]. Improved survival rates following the implementation of cyclophosphamide-based treatment regimens [11] have made the long-term effects of neutrophilic inflammation on the GPA lung more pertinent. The mechanisms and mediators associated with neutrophil recruitment to the lung in GPA are undetermined.

Cytokines play an important regulatory role in neutrophil migration to sites of inflammation [12]. Upregulated cytokine concentrations have been implicated in the neutrophilia associated with acute lung injury and pulmonary fibrosis,

\section{AFFLIATIONS}

*Lung Injury and Fibrosis Treatment Programme, Dept of Medical Sciences,

'Dept of Renal Immunobiology, Division of Infection and Immunity, Medical School, University of Birmingham, Birmingham, and

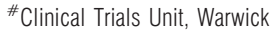
Medical School, University of Warwick, Coventry, UK.

\section{CORRESPONDENCE}

D.R. Thickett

Lung Investigation Unit

1st floor

Nuffield House

Queen Elizabeth Hospital

Birmingham

B15 2TH

UK

E-mail: d.thickett@bham.ac.uk

Received:

Oct 142010

Accepted after revision:

March 042011

First published online:

Sept 012011 
by promoting chemotaxis within the alveolar compartment [13, 14]. Previous small studies have suggested increased levels of neutrophilic chemokines $[15,16]$ in the serum of GPA patients and elevated CXC chemokine ligand (CXCL) 8 in the glomeruli of patients with active GPA [17]. Neutrophilic chemokine levels have not been established within the lung, although a case report has reported an increase in CXCL8, interleukin (IL)$1 \beta$ and granulocyte colony-stimulating factor (G-CSF) [18].

The aims of this study were to determine the dynamics of neutrophilic inflammation during acute and relapsing GPA, its relationship to neutrophilic chemokines with BALF, and the functionally important chemokines in GPA BALF. Comparisons were made with idiopathic pulmonary fibrosis (IPF) and normal controls. IPF patients were recruited as disease controls because of the well described pulmonary neutrophilia associated with the disorder. The chemotactic potential of BALF was examined using the under-agarose method, and specific antibodies against CXCL8 and IL-1 $\beta$.

\section{MATERIALS AND METHODS \\ Recruitment}

26 patients with GPA were recruited from the University Hospital Birmingham (Birmingham, UK) between 2003 and 2006. Active patients were sequentially recruited on admission if bronchoscopy was deemed safe according to local guidelines. Remission patients were sequentially enrolled from the vasculitis clinic at routine clinic appointments. All patients complied with the American College of Rheumatology criteria and the Chapel Hill consensus statement definition of GPA [19]. Patients were assessed for disease activity at the time of inclusion using the Birmingham Vasculitis Activity Score (BVAS), a validated index that comprises a weighted item list, which assesses activity attributable to active vasculitis [20]. Remission was defined as a BVAS of less than one and active patients had a score greater than four. Where there was a suggestion of disease relapse with negative ANCA, tissue biopsy confirmed the presence of active vasculitis in all cases. The Vasculitis Damage Index assessed severity of irreversible injury in specific organ systems.

IPF patients were sequentially recruited from a research interstitial lung disease clinic and diagnosed according to American Thoracic Society/European Respiratory Society guidelines. Healthy controls who were free from respiratory disease were recruited from hospital volunteers. The local research ethics committee gave approval for the study and informed, written consent was obtained (ref. 2003/166).

\section{Radiology}

All GPA and IPF patients underwent high-resolution computed tomography (HRCT) of the chest. Scans were read blinded to conditions by a pulmonologist (D.R. Thickett) and a radiologist (P. Gair, University Hospital Birmingham) with an interest in interstitial lung disease, and were assessed for the presence or absence of bronchiectasis, fibrosis, cavities, nodules and emphysema, features previously noted in a review of pulmonary findings in GPA [1].

\section{Pulmonary function testing}

Forced vital capacity was measured in GPA and IPF patients using the Jaeger Compact system (Viasys Healthcare; Carefusion, Basingstoke, UK). Transfer capacity of the lung for carbon monoxide was measured using the single-breath technique. Data are presented as \% predicted.

\section{Bronchoalveolar lavage}

All patients underwent bronchoalveolar lavage according to national guidelines [2]. Bronchoscopy was performed according to a standardised method. $180 \mathrm{~mL}$ (three $60-\mathrm{mL}$ syringes) sterile $0.9 \%$ saline was instilled into the right middle lobe bronchus and manually aspirated. BALF was filtered through coarse surgical gauze to remove mucous debris and centrifuged at $500 \times g$ for $6 \mathrm{~min}$ at $4^{\circ} \mathrm{C}$. The supernatant was removed and frozen at $-80^{\circ} \mathrm{C}$ for subsequent analysis. The cell pellet was resuspended in RPMI 1640 medium (Sigma-Aldrich, Poole, UK). A cell count was performed using a haemocytometer and cell differentials analysed following staining (Diff-Quick) of a cytospin slide.

\section{Measurements}

BALF IL-1 $\beta$, CXCL8, epithelial neutrophil-activating protein (ENA)-78, G-CSF and granulocyte-macrophage colony-stimulating factor (GM-CSF) levels were measured by ELISA (R\&D Systems, Abingdon, UK) according to the manufacturer's instructions. These assays had an average coefficient of variance of $8 \%$ (range 6.6-9.6\%). MPO activity was measured using a colorimetric substrate assay [21].

\section{Under-agarose chemotaxis}

Normal neutrophils were purified from peripheral blood using discontinuous Percoll ${ }_{\circledR}$ gradients [22] (Sigma-Aldrich). A $>98 \%$ cell purity and viability was confirmed using a cytospin. The chemotactic activity of BALF on control neutrophils was measured using the under-agarose method, as previously described [14, 24]. The same donor control was used for all neutrophil experiments. In short, agarose plates with imprinted wells were concentrically filled with the chemotactic ligand, the neutrophil suspension $\left(5 \times 10^{7}\right.$ cells $\left.\cdot \mathrm{mL}^{-1}\right)$ and an appropriate control. The plates were incubated for $2 \mathrm{~h}$ to allow migration, fixed with glutaraldehyde and then stained with Azure B stain. The furthest point of neutrophil migration towards the chemoattractant and control was measured in millimetres by two individuals, aided by a graded graticule, blinded to the diagnosis or chemoattractant. Chemotaxis was measured as the limit of migration of neutrophils towards the outer chemoattractant ring, and chemokinesis as the activation of neutrophils and subsequent random migration towards the inner control ring. Net chemotaxis describes the difference between the two. Apart from the initial BALF experiments, where the measurements are individually displayed, the data are presented as net chemotaxis (in millimetres).

For all chemotaxis experiments, a positive $\left(10^{-7} \mathrm{M}\right.$ formyl-MetLeu-Phe (fMLP)) and negative ( $0.9 \%$ saline) chemoattractant control were added, and experiments with $>10 \%$ interassay variability were repeated. Experiments were performed in triplicate for each tested condition.

For the cytokine blocking experiments, BALF was pre-incubated for $20 \mathrm{~min}$ with anti-IL8 antibody $\left(1 \times 10^{-7} \mathrm{M}\right)$, anti-IL1 $\beta$ antibody $\left(2 \times 10^{-7} \mathrm{M}\right)$ (both R\&D Systems) and a CXC chemokine receptor (CXCR)2 antagonist (SB225002; $1 \times 10^{-8} \mathrm{M}$ ). Optimum inhibitor concentrations were established in preliminary experiments (data not shown). The effect of the antibodies on chemotaxis was 
examined to show that their actions were not due to nonspecific binding (data not shown).

\section{Analysis}

The chemotaxis data are presented as mean \pm SE and betweendisease-cohort comparisons were made using ANOVA with Tukey's post hoc analysis. All other data were nonparametric and presented as median (interquartile range (IQR)). IQR describes the difference between the lower and upper quartiles. Differences between the disease groups in cell counts and cytokines were explored using a Kruskal-Wallis test with Dunn's test for between-group comparison. Correlations between chemotaxis and cytokines were made using Spearman's rank test. All statistical analyses were performed using SPSS version 15 (IBM, Portsmouth, UK). A p-value $\leqslant 0.05$ was considered statistically significant.

\begin{tabular}{|c|c|c|c|c|}
\hline & \multicolumn{2}{|c|}{ GPA } & \multirow[t]{2}{*}{ IPF } & \multirow[t]{2}{*}{ Normal } \\
\hline & Active & Remission & & \\
\hline Patients $\mathbf{n}$ & 19 & 7 & 20 & 6 \\
\hline Males & 52 & 42 & 77 & 33 \\
\hline Age yrs & $56.6 \pm 28.25$ & $51.0 \pm 18.8$ & $70 \pm 18.8$ & $45 \pm 13.8$ \\
\hline \multicolumn{5}{|l|}{ Smoking } \\
\hline $\begin{array}{l}\text { Never-/ex-/current } \\
\text { smokers n }\end{array}$ & $11 / 13 / 1$ & $3 / 3 / 0$ & $3 / 20 / 3$ & $2 / 3 / 121$ \\
\hline Pack-yrs & $10.1 \pm 24.3$ & $6.8 \pm 14.0$ & $30 \pm 26.2$ & \\
\hline FVC \% pred & $113.0 \pm 23.5$ & $108.5 \pm 26.0$ & $69.5 \pm 27.8$ & NA \\
\hline TL,CO \% pred & $85.0 \pm 11.0$ & $86.0 \pm 31.0$ & $49.0 \pm 13.0$ & NA \\
\hline CRP & $23.3 \pm 32.1$ & $10.0 \pm 40.1$ & $6 \pm 10.3$ & $<4$ \\
\hline ANCA positive & 92 & 33 & 0 & 0 \\
\hline PR3 positive & 80 & 44 & 0 & 0 \\
\hline MPO positive & 12 & 0 & 0 & 0 \\
\hline BVAS & $4.5 \pm 9.0$ & $0.0 \pm 2.4$ & NA & NA \\
\hline VDI & $4.2 \pm 0.6$ & $3.8 \pm 0.8$ & NA & NA \\
\hline Bronchiectasis & 30 & 50 & $74^{\circ}$ & NA \\
\hline Interstitial changes ${ }^{\#}$ & 54 & 33 & 100 & NA \\
\hline Cavities & 36 & 17 & 0 & NA \\
\hline Nodules & 82 & 66 & 0 & NA \\
\hline Haemoptysis & 12 & 0 & 0 & NA \\
\hline Steroids & 78 & 77 & 0 & 0 \\
\hline Cyclophosphamide & 43 & 11 & 0 & 0 \\
\hline Azathioprine & 17 & 44 & 0 & 0 \\
\hline Mycophenolate & 9 & 33 & 0 & 0 \\
\hline Methotrexate & 0 & 11 & 0 & 0 \\
\hline Rituximab/infliximab & 9 & 0 & 0 & 0 \\
\hline No medication & 13 & 22 & 0 & 0 \\
\hline
\end{tabular}

Data are presented as $\%$ or mean $\pm \mathrm{SE}$, unless otherwise stated. GPA: granulomatosis with polyanglitis (Wegener's); IPF: idiopathic pulmonary fibrosis; FVC: forced vital capacity; \% pred: \% predicted; $T L, C O$ : transfer capacity of the lung for carbon monoxide; CRP: C-reactive protein; ANCA: antineutrophil cytoplasmic antibody; PR: proteinase; MPO: myeloperoxidase; BVAS: Birmingham Vasculitis Activity Score; VDI: Vasculitis Damage Index; NA: not applicable. ${ }^{\#}$ : included scarring, fibrosis and atelectasis; ": traction bronchiectasis.

\section{RESULTS}

\section{Demographics}

19 GPA patients with a BVAS greater than four were classified as active. One patient had a BVAS of three with biopsy evidence of relapse and was included in the active group. Seven GPA patients were recruited and deemed to be clinically in remission. $20 \mathrm{IPF}$ patients and six normal controls were recruited. Smoking history and the presence of chronic lung damage, as evidenced by pulmonary function testing and chest HRCT, did not predict the neutrophil count (data not shown). Serology and HRCT findings expressed as percentage of the group are presented in table 1.

\section{Neutrophilic inflammation and activation is persistent in GPA patients}

Median (IQR) BALF neutrophil counts were higher in the active (5.0 (27.0)\%; $p=0.007)$ and remission (8.0 (60.9)\%; $\mathrm{p}=0.072)$ GPA patients compared with normal controls $(1.8$ (2.3)\%). IPF BALF neutrophil counts were higher than those in normal controls $(15.3(26.8) \%$; $<<0.001)$ but not significantly different from the active $(p=0.210)$ or remission $(p=0.855)$ GPA groups (fig. 1a).

The median (IQR) total cell count was significantly increased in the active and remission GPA groups $\left(108.0 \times 10^{4}\left(159.0 \times 10^{4}\right)\right.$ and $74.2 \times 10^{4}\left(170.4 \times 10^{4}\right)$ cells $\left.\cdot \mathrm{mL}^{-1}\right)$ compared with normal controls $\left(3.8 \times 10^{4}\left(49.9 \times 10^{4}\right)\right.$ cells $\cdot \mathrm{mL}^{-1} ; \mathrm{p}=0.004$ and $\mathrm{p}=0.019$, respectively). The total cell count was higher in the IPF cohort $\left(123.0 \times 10^{4}\right.$ $\left(237.0 \times 10^{4}\right)$ cells $\left.\cdot \mathrm{mL}^{-1}\right)$ than in normal controls $(p=0.001)$ but there was no difference with either GPA activity group.

There was increased median (IQR) MPO activity in the active (0.064 (0.403) U. $\left.\mathrm{mL}^{-1} ; \mathrm{p}<0.001\right)$ and remission $(0.122(0.500)$ $\left.\mathrm{U} \cdot \mathrm{mL}^{-1} ; \mathrm{p}=0.022\right)$ GPA groups compared with normal controls (0.001 (0.003) U.mL $\mathrm{m}^{-1}$ ) (fig. 1b). IPF BALF MPO activity $(0.096$ $\left.(0.244) \mathrm{U} \cdot \mathrm{mL}^{-1}\right)$ was elevated compared with normal controls $(p<0.001)$ but there was no difference compared with either GPA activity group (fig. 1b). BALF neutrophils and MPO activity correlated in the active GPA group $(\mathrm{r}=0.484, \mathrm{p}=0.005)$ and IPF $(r=0.563, p=0.002)$, but not in the remission GPA group $(r=0.069$, $\mathrm{p}=0.832)$.

There were no relationships between neutrophil count, MPO and lung function in GPA, but the BALF neutrophil count did correlate with serum $C$-reactive protein $(\mathrm{r}=0.460, \mathrm{p}=0.016)$.

\section{BALF and plasma cytokine levels}

There were increased concentrations of BALF CXCL8 and ENA-78 in both the active and remission GPA patients, and IPF patients, compared with normal controls (table 2). There was no difference in IL-1 $\beta$, G-CSF and GM-CSF concentrations between cohorts.

Plasma ENA-78 concentrations were elevated, and IL-1 $\beta$, GCSF and GM-CSF were reduced in the active GPA group compared with normal controls. IL- $1 \beta$ concentrations were lower in the remission GPA group compared with controls. In contrast to the BALF, there was no difference in the plasma concentrations of CXCL8 in active or remission GPA patients compared with controls (table 3 ). There were increased plasma concentrations of CXCL8 and ENA-78 in IPF patients compared with controls, but lower concentrations of IL-1 $\beta$, G-CSF and GM-CSF. 

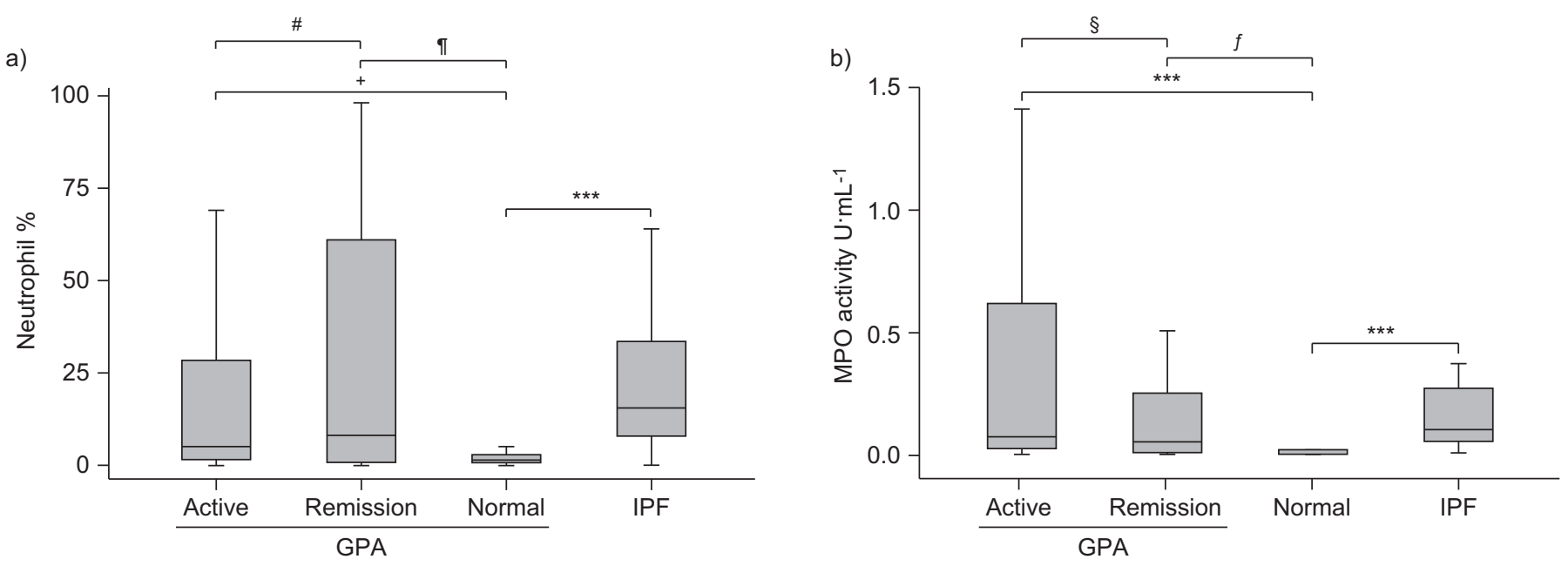

FIGURE 1. a) Bronchoalveolar lavage fluid neutrophil count expressed as a percentage of total cells and b) myeloperoxidase (MPO) activity measured by colorimetric assay. Data are presented as median (interquartile range). GPA: granulomatosis with polyangiitis (Wegener's); IPF: idiopathic pulmonary fibrosis. " $: p=0.624 ; ": p=0.072$ ${ }^{+}: p=0.007{ }^{\text {s }}: p=0.428 ;{ }^{f}: p=0.022 ;{ }^{* * *}: p<0.001$.

\section{The effect of BALF on neutrophil chemotaxis and chemokinesis}

Median (IQR) eutrophil chemotaxis towards BALF from the active (6.16 (3.67) $\mathrm{mm} ; \mathrm{p}=0.04)$ and remission $(8.43$ (2.34) $\mathrm{mm}$; $\mathrm{p}=0.003)$ GPA groups, and IPF patients (8.71 (3.71) mm; $\mathrm{p}=0.002)$ was increased compared with normal BALF (6.5 $(1.21) \mathrm{mm})$. There was no difference in chemotaxis between either GPA group and IPF ( $p=0.451)$. Active (6.8 (1.53) min; $\mathrm{p}=0.041)$ and remission $(7.37(2.23) \mathrm{mm} ; \mathrm{p}=0.007) \mathrm{GPA}$, and IPF BALF (7.62 (3.71) mm; p=0.04) also exhibited increased neutrophil chemokinesis compared with normal control BALF $(5.62(1.33) \mathrm{mm})$. There was a close correlation between chemokinesis and chemotaxis in GPA $(r=0.941, p<0.001)$ and IPF $(\mathrm{r}=0.968, \mathrm{p}<0.001)$.

The median (IQR) net chemotaxis of active GPA BALF (1.33 (1.17) $\mathrm{mm}$ ) and IPF BALF (1.50 (1.09) mm) was elevated compared with normal controls $(0.33(0.67) \mathrm{mm})$ (fig. 2). The net chemotaxis of GPA remission BALF (0.67 (1.25) mm) was lower than the active group and higher than normal controls, but was not significantly different from either group. The net chemotactic potential of GPA BALF correlated with the measured BALF neutrophil count $(\mathrm{r}=0.567, \mathrm{p}=0.004)$ and MPO activity $(r=0.672, p=0.012)$. There was no such relationship in the IPF patients' BALF. BALF chemotactic potential did not relate to the presence of a significant bacterial culture from BALF in either active or remission groups (data not shown).

\section{Investigation of a role for IL-1 $\beta$ and CXCL8 in GPA BALF- induced chemotaxis}

The amount of BALF-induced chemotaxis correlated with BALF CXCL8 ( $r=0.640, p=0.001)$ and IL-1 $\beta(r=0.761, p=0.001)$, but not with GM-CSF ( $\mathrm{r}=0.171, \mathrm{p}=0.413)$, G-CSF $(\mathrm{r}=0.291$, $\mathrm{p}=0.157)$ or ENA-78 $(\mathrm{r}=0.148, \mathrm{p}=0.322)$ levels. As a consequence of these results, specific antibodies were used to explore the effects of CXCL8, IL-1 $\beta$ and a CXCR2 antagonist (SB225002) on GPA BALF-induced chemotaxis.

The chemotactic response to active GPA BALF $(n=9)$ (mean \pm SE $4.24 \pm 0.39 \mathrm{~mm}$ ) was inhibited by anti-CXCL8

\section{TABLE 2 Bronchoalveolar lavage fluid cytokine levels}

\begin{tabular}{|c|c|c|c|c|}
\hline \multirow[t]{2}{*}{ Cytokine } & \multicolumn{2}{|c|}{ GPA } & \multirow[t]{2}{*}{ IPF } & \multirow[t]{2}{*}{ Normal } \\
\hline & Active & Remission & & \\
\hline $\mathrm{CXCL8} \mathrm{pg} \cdot \mathrm{mL}^{-1}$ & $422.8(314.0)^{*}$ & $2725.0(13527.9)^{\star}$ & $329.0(868.3)^{*}$ & $30.5(58.0)$ \\
\hline $\mathrm{IL}-1 \beta \mathrm{pg} \cdot \mathrm{mL}^{-1}$ & $4.0(41.5)$ & 74.9 (306.3) & $18.6(41.1)$ & $3.8(1.6)$ \\
\hline ENA-78 $\mathrm{pg} \cdot \mathrm{mL}^{-1}$ & $259.3(761.3)^{\star}$ & $153.4(263.5)^{*}$ & $325.7(475.4)^{*}$ & $7.3(41.4)$ \\
\hline
\end{tabular}

Data are presented as median (interquartile range). There were no significant differences between disease cohorts. All cytokine concentrations were measured by ELISA (R\&D Systems, Abingdon, UK). GPA: granulomatosis with polyangiitis (Wegener's); IPF: idiopathic pulmonary fibrosis; CXCL: CXC chemokine ligand; IL: interleukin; ENA: epithelial neutrophil-activating protein; G-CSF: granulocyte colony-stimulating factor; GM-CSF: granulocyte-macrophage colony-stimulating factor. *: p<0.05 compared with normal controls when compared using Kruskal-Wallis and Dunn's tests 


\section{TABLE 3 Plasma cytokine levels}

\begin{tabular}{|c|c|c|c|c|}
\hline \multirow[t]{2}{*}{ Cytokine } & \multicolumn{2}{|c|}{ GPA } & \multirow[t]{2}{*}{ IPF } & \multirow[t]{2}{*}{ Normal } \\
\hline & Active & Remission & & \\
\hline $\mathrm{IL}-1 \beta \mathrm{pg} \cdot \mathrm{mL}^{-1}$ & $0.0(0.0)^{*}$ & $0.0(0.0)^{*}$ & $0.0(0.1)^{*}$ & $8.6(3.9)$ \\
\hline ENA-78 $\mathrm{pg} \cdot \mathrm{mL}^{-1}$ & $1009.7(828.1)^{*}$ & 723.0 (937.8) & $984.5(1209.0)^{\star}$ & $151.7(187.0)$ \\
\hline
\end{tabular}

$(1.5 \pm 0.24 \mathrm{~mm} ; \mathrm{p}=0.001)$, anti-IL-1 $\beta(1.26 \pm 0.22 \mathrm{~mm} ; \mathrm{p}<0.001)$ and the CXCR2 antagonist $(0.98 \pm 0.25 \mathrm{~mm} ; \mathrm{p}=0.001)$. The response to BALF was further inhibited by the combination of anti-IL-1 $\beta$ and anti-CXCL8 (0.39 $\pm 0.15 \mathrm{~mm}$; $\mathrm{p}=0.001)$ (fig. 3).

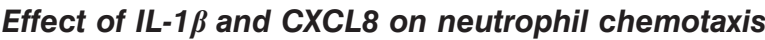

IL-1 $\beta$ resulted in a dose-dependent increase in normal neutrophil chemotaxis $\left(20,200\right.$ and $2,000 \mathrm{pg} \cdot \mathrm{mL}^{-1} \mathrm{IL}-1 \beta$ gave $2.3,4.0$ and $11.0 \mathrm{~mm}$ chemotaxis, respectively), which could be abrogated to $90 \%$ chemotaxis by anti-IL-1 $\beta\left(2.5 \times 10^{-7} \mathrm{M}\right)$ $(\mathrm{p}<0.001)$. CXCL8 caused a dose-dependent increase in chemotaxis $\left(10^{-9}, 10^{-8}, 10^{-7}\right.$ and $10^{-6} \mathrm{M}$ gave $0.7,3.7,5.7$ and $9.3 \mathrm{~mm}$ chemotaxis, respectively), which could be inhibited by anti-CXCL8 $\left(2 \times 10^{-7} \mathrm{M}\right)(\mathrm{p}<0.001)$. There was a synergistic chemotactic response when IL-1 $\beta\left(200 \mathrm{pg} \cdot \mathrm{mL}^{-1}\right)$ and CXCL8

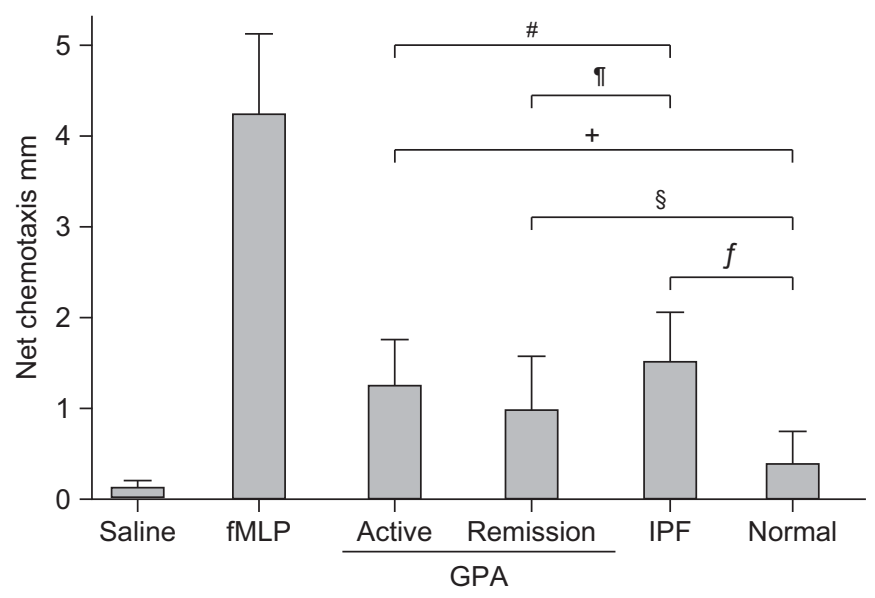

FIGURE 2. Chemotactic response of neutrophils to bronchoalveolar lavage fluid (BALF). The under-agarose method was used to measure the distance migrated by normal neutrophils towards either BALF or saline control. Net chemotaxis describes the difference between migration towards the chemoattractant and control. Data were examined using ANOVA with Tukey's post hoc analysis. Chemotaxis was measured with all BALF samples. Data are presented as mean \pm SE. fMLP: formyl-Met-Leu-Phe; GPA: granulomatosis with polyangiitis (Wegener's); IPF: idiopathic pulmonary fibrosis. \#: $p=0.546$; $\because p=0.161$; $+: \mathrm{p}=0.006 ;{ }^{\varsigma}: \mathrm{p}=0.077 ;$ : $\mathrm{p}=0.001$. $\left(10^{-7} \mathrm{M}\right)$ were co-incubated (IL-1 $4.0 \mathrm{~mm}$, CXCL8 $5.7 \mathrm{~mm}$, and IL-1 $\beta$ plus CXCL8 $12.0 \mathrm{~mm}$; ANOVA $\mathrm{p}=0.013)$. The antibodies did not influence fMLP-stimulated chemotaxis (fig. 4).

\section{DISCUSSION}

This large bronchoscopy study has confirmed the findings of previous studies reporting BALF neutrophilia during active GPA disease $[8,25]$ and one small series that demonstrated increased neutrophils during remission [9]. Elevated MPO levels indicated that these neutrophils were activated. BALF concentrations of CXCL8 and ENA-78, but not IL-1 $\beta$, G-CSF and GM-CSF, were elevated in both active and remission GPA patients compared with normal controls. BALF from GPA patients was found to stimulate neutrophil chemotaxis compared with normal controls, and this was inhibited by antibodies against CXCL8 and IL-1 $\beta$, and a CXCR2 antagonist.

The presence of a neutrophilia has particular relevance to GPA due to the strong association of the disease with ANCAs [25]. The molecular mechanisms contributing to the BALF neutrophilia seen in GPA are not well understood. Leukocyte scanning of vasculitis patients has shown that GPA patients have increased neutrophil margination in the lung [26]. Recruitment of neutrophils to the tissues is a multistep process that is partially directed by cytokines. The BALF neutrophil count correlated with CXCL8 and IL-1 $\beta$, which may suggest a role for cytokines in neutrophil recruitment in GPA. This finding was supported by the BALF experiments, which found that BALF-induced chemotaxis was inhibited by specific antibodies against CXCL8 and IL-1 $\beta$. The degree of inhibition that resulted from incubation with these antibodies suggested that CXCL8 and IL-1 $\beta$ were the major cytokine determinants of BALF-induced chemotaxis.

Plasma CXCL8 levels were elevated in GPA and IPF patients compared with normal controls, albeit at a 10-fold lower level than in the BALF, suggesting local lung production. CXCL8 is known to have potent chemotactic activity and has an important role in the accumulation of neutrophils in the lungs of patients with IPF [27] and adult respiratory distress syndrome [28], where persistent neutrophilia is associated with mortality. CXCL8 is able to increase neutrophil adhesiveness and upregulate intercellular adhesion molecule-1 


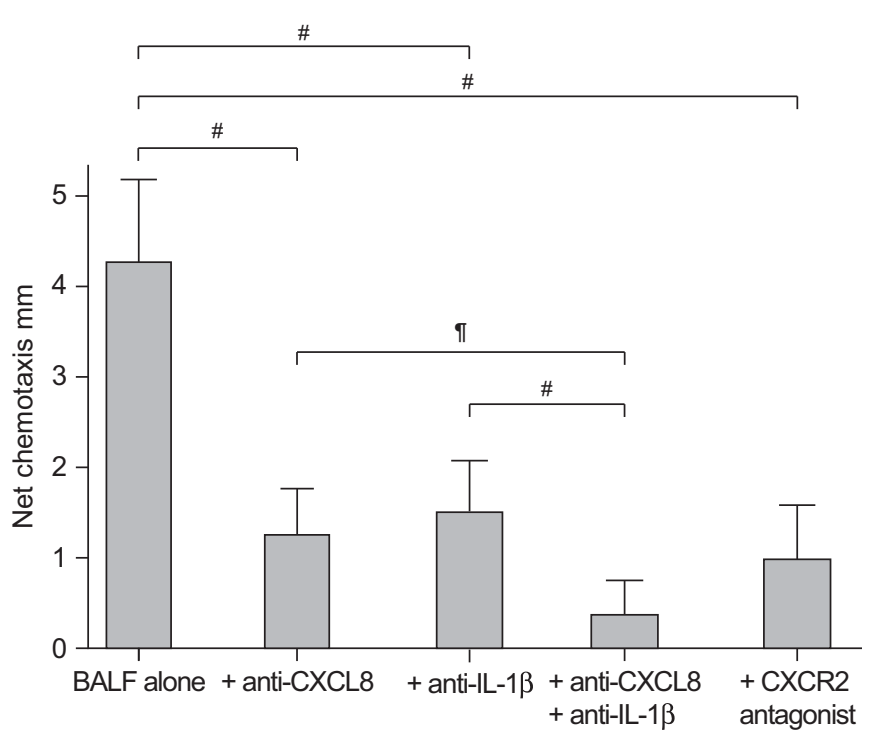

FIGURE 3. The effect of anti-interleukin (IL)-1 $\beta$ and anti-CXC chemokine ligand (CXCL)8 on bronchoalveolar lavage fluid (BALF)-induced chemotaxis. Chemotaxis to active granulomatosis with polyangiitis (Wegener's) (GPA) BALF was inhibited by anti-IL-1 $\beta\left(2 \times 10^{-7} \mathrm{M}\right)$, anti-CXCL8 $\left(10^{-6} \mathrm{M}\right)$ and a CXC chemokine receptor (CXCR)2 antagonist (SB225002; $\left.1 \times 10^{-8} \mathrm{M}\right)(\mathrm{p}<0.001$ for each experiment). The combination of anti-IL-1 $\beta$ and anti-CXCL8 further inhibited the chemotactic response. All experiments were performed on nine active GPA BALF samples and repeated in triplicate. ${ }^{\#}: p=0.001 ;{ }^{\circ}: p=0.002$.

expression, potentially promoting margination [16]. Aside from neutrophil recruitment, CXCL8 has other mechanisms relevant to GPA, with ANCAs directly stimulating CXCL8 release from both monocytes and neutrophils [17, 29]. CXCL8 and other cytokines, including tumour necrosis factor- $\alpha$, further stimulate translocation of PR3 to the cell surface, increasing the likelihood of ANCA binding [30, 31]. CXCL8 exerts its actions through the CXCR1 and CXCR2 receptors $[32,33]$, and our experiments suggest a role for the CXCR2 receptor in BALF-induced chemotaxis.

While the role of CXCL8 as a neutrophil chemoattractant is well established, the role of IL- $\beta$ remains controversial, although it has established actions on neutrophil adherence $[12,34]$ and induces a number of cells to produce known neutrophil chemoattractants, including CXCL8 [35]. ENA-78 is a potent neutrophil chemotactant that has been found to be upregulated in inflammatory conditions, such as rheumatoid arthritis [36], but although we found increased levels in GPA BALF, there was no significant relationship with the BALF neutrophilia. Haematopoietic factors regulate neutrophil production and maturation in the bone marrow. This study has shown increased levels of G-CSF, a chemokine which has established actions on the proliferation and activation of neutrophils [37], in GPA BALF. GM-CSF is able to stimulate bone marrow precursors to proliferate and differentiate into neutrophil cell lines [9]. GM-CSF, but not G-CSF, is able to upregulate PR3 expression on neutrophils and, thus, has specific relevance to GPA pathology [38]; however, concentrations were not significantly elevated in our GPA cohort. Neutrophil chemokines were also elevated in BALF from IPF

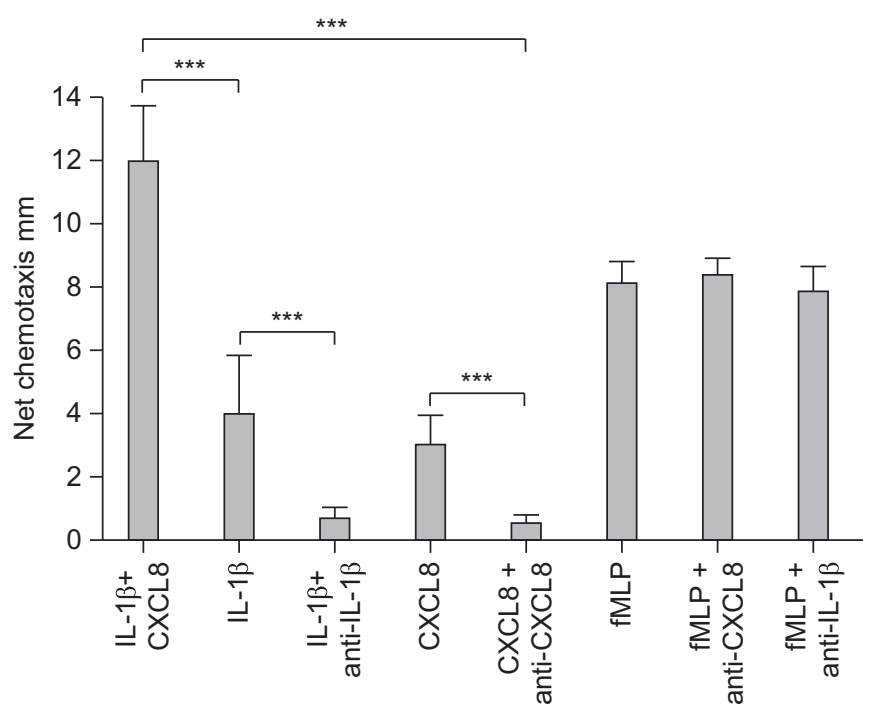

FIGURE 4. The effect of interleukin (IL)-1 $\beta$ and CXC chemokine ligand $(\mathrm{CXCL}) 8$, and their specific antibodies upon neutrophil chemotaxis. A chemotactic response was demonstrated to $\mathrm{LL}-1 \beta\left(200 \mathrm{pg} \cdot \mathrm{mL}^{-1}\right)$ and CXCL8 $\left(10^{-7} \mathrm{M}\right)$. There was enhanced chemotactic activity when $\mathrm{IL}-1 \beta$ and CXCL8 were co-incubated. The effects of IL-1 $\beta$ and CXCL8 were reduced by specific antibodies, which did not affect formyl-Met-Leu-Phe (fMLP)-stimulated chemotaxis. Data are presented as mean \pm SE. ${ }^{* * *}: \mathrm{p}<0.001$.

patients, but only in patients with GPA did CXCL8 and IL-1 $\beta$ relate to BALF neutrophil counts.

The consequences of persistent neutrophilic inflammation and unopposed protease action are best understood in chronic obstructive airways disease and bronchiectasis [10], features of which have been reported in both IPF and GPA cohorts [7]. Elevated MPO activity has previously been reported in active GPA [39]; this study confirms these findings and goes on to show that MPO activity remains elevated during clinical remission. Whether this reflects ongoing neutrophil activation secondary to disease activity or chronic lung damage and infection is unknown. We have previously reported that pathogens are commonly grown from both GPA and IPF BALF [40]; however, bacterial growth was not associated with a higher neutrophil count or BALF chemotactic potential.

There are a number of limitations to this study. First, the under-agarose method cannot fully reflect the complexities of the human lung. However, the degree of neutrophil chemotaxis correlated with percentage neutrophil count and MPO levels in the BALF, supporting our hypothesis that this model does, to some extent, reflect in vivo dynamics. Also, there was significant variability between under-agarose experiments performed at different time-points, as seen in the different graphs. For this reason, each set of experiments was performed on the same day, and each sample and control was run in triplicate. Secondly, it cannot necessarily be extrapolated that undiluted epithelial lining fluid (ELF) would give similar results to the BALF. Repeating these experiments using a different method to assess chemotaxis with directly sampled ELF, and evaluating other cytokines and cytokine receptors, would allow this research to be taken forward. Additionally, 
examining tissue samples would confirm whether these chemokines are also elevated in the interstitium. Finally, the median BVAS was lower in the acute patients than had been expected from other studies [7]. Our interpretation is that a number of patients had respiratory-limited GPA and that the BVAS, which is weighted towards renal disease, does not score single-organ pulmonary disease highly.

In conclusion, this study provides further evidence that neutrophilic inflammation is a key manifestation of the GPA lung during disease activity and during remission. We demonstrate elevated alveolar cytokine concentrations, and evidence that CXCL8 and IL-1 $\beta$ may provide a continuous stimulus for neutrophil chemotaxis. The mechanisms of action of cytokines in the neutrophil chemotactic response warrant further investigation, as targeting pathways resulting in the activation and recruitment of neutrophils may offer alternative therapeutic options.

\section{STATEMENT OF INTEREST}

None declared.

\section{REFERENCES}

1 Gross WL, Csernok E, Flesch BK. "Classic" anti-neutrophil cytoplasmic autoantibodies (cANCA), "Wegener's autoantigen" and their immunopathogenic role in Wegener's granulomatosis. J Autoimmun 1993; 6: 171-184.

2 Falk RJ, Terrell RS, Charles LA, et al. Anti-neutrophil cytoplasmic autoantibodies induce neutrophils to degranulate and produce oxygen radicals in vitro. Proc Natl Acad Sci USA 1990; 87: $4115-4119$.

3 Xiao $\mathrm{H}$, Heeringa $\mathrm{P}, \mathrm{Hu} \mathrm{P}$, et al. Antineutrophil cytoplasmic autoantibodies specific for myeloperoxidase cause glomerulonephritis and vasculitis in mice. J Clin Invest 2002; 110: 955-963.

4 Brouwer E, Huitema MG, Mulder AH, et al. Neutrophil activation in vitro and in vivo in Wegener's granulomatosis. Kidney Int 1994; 45: 1120-1131.

5 Cordier JF, Valeyre D, Guillevin L, et al. Pulmonary Wegener's granulomatosis. A clinical and imaging study of 77 cases. Chest 1990; 97: 906-912.

6 Schnabel A, Reuter M, Csernok E, et al. Subclinical alveolar bleeding in pulmonary vasculitides: correlation with indices of disease activity. Eur Respir J 1999; 14: 118-124.

7 Newall C, Schinke S, Savage CO, et al. Impairment of lung function, health status and functional capacity in patients with ANCAassociated vasculitis. Rheumatology (Oxford) 2005; 44: 623-628.

8 Schnabel A, Csernok E, Braun J, et al. Activation of neutrophils, eosinophils, and lymphocytes in the lower respiratory tract in Wegener's granulomatosis. Am J Respir Crit Care Med 2000; 161: 399-405.

9 Hoffman GS, Sechler JM, Gallin JI, et al. Bronchoalveolar lavage analysis in Wegener's granulomatosis. A method to study disease pathogenesis. Am Rev Respir Dis 1991; 143: 401-407.

10 Amitani R, Wilson R, Rutman A, et al. Effects of human neutrophil elastase and Pseudomonas aeruginosa proteinases on human respiratory epithelium. Am J Respir Cell Mol Biol 1991; 4: 26-32.

11 Guillevin L, Cordier JF, Lhote F, et al. A prospective, multicenter, randomized trial comparing steroids and pulse cyclophosphamide versus steroids and oral cyclophosphamide in the treatment of generalized Wegener's granulomatosis. Arthritis Rheum 1997; 40: 2187-2198.

12 Wagner JG, Roth RA. Neutrophil migration mechanisms, with an emphasis on the pulmonary vasculature. Pharmacol Rev 2000; 52: 349-374.
13 Strieter RM, Gomperts BN, Keane MP. The role of CXC chemokines in pulmonary fibrosis. J Clin Invest 2007; 117: 549-556.

14 Perkins GD, Nathani N, McAuley DF, et al. In vitro and in vivo effects of salbutamol on neutrophil function in acute lung injury. Thorax 2007; 62: 36-42.

15 Torheim EA, Yndestad A, Bjerkeli V, et al. Increased expression of chemokines in patients with Wegener's granulomatosis modulating effects of methylprednisolone in vitro. Clin Exp Immunol 2005; 140: 376-383.

16 Ohta N, Fukase S, Aoyagi M. Serum levels of soluble adhesion molecules ICAM-1, VCAM-1 and E-selectin in patients with Wegener's granulomatosis. Auris Nasus Larynx 2001; 28: 311-314.

17 Cockwell P, Brooks CJ, Adu D, et al. Interleukin-8: a pathogenetic role in antineutrophil cytoplasmic autoantibody-associated glomerulonephritis. Kidney Int 1999; 55: 852-863.

18 Mukae H, Matsumoto N, Ashitani J, et al. Neutrophil-related cytokines and neutrophil products in bronchoalveolar lavage fluid of a patient with ANCA negative Wegener's granulomatosis. Eur Respir J 1996; 9: 1950-1954.

19 Jennette JC, Falk RJ, Andrassy K, et al. Nomenclature of systemic vasculitides. Proposal of an international consensus conference. Arthritis Rheum 1994; 37: 187-192.

20 Luqmani RA, Bacon PA, Moots RJ, et al. Birmingham Vasculitis Activity Score (BVAS) in systemic necrotizing vasculitis. QJM 1994; 87: 671-678.

21 Perkins GD, Chatterjie S, McAuley DF, et al. Role of nonbronchoscopic lavage for investigating alveolar inflammation and permeability in acute respiratory distress syndrome. Crit Care Med 2006; 34: 57-64.

22 Giudicelli J, Philip PJ, Delque P, et al. A single-step centrifugation method for separation of granulocytes and mononuclear cells from blood using discontinuous density gradient of Percoll. J Immunol Methods 1982; 54: 43-46.

23 Heit B, Kubes P. Measuring chemotaxis and chemokinesis: the under-agarose cell migration assay. Sci STKE 2003; 2003: L5.

24 Schnabel A, Reuter M, Gloeckner K, et al. Bronchoalveolar lavage cell profiles in Wegener's granulomatosis. Respir Med 1999; 93: 498-506.

25 Schnabel A, Csernok E, Braun J, et al. Inflammatory cells and cellular activation in the lower respiratory tract in Churg-Strauss syndrome. Thorax 1999; 54: 771-778.

26 Jonker ND, Peters AM, Gaskin G, et al. A retrospective study of radiolabeled granulocyte kinetics in patients with systemic vasculitis. J Nucl Med 1992; 33: 491-497.

27 Cantin AM, North SL, Fells GA, et al. Oxidant-mediated epithelial cell injury in idiopathic pulmonary fibrosis. J Clin Invest 1987; 79: 1665-1673.

28 Cailes JB, O'Connor C, Pantelidis $\mathrm{P}$, et al. Neutrophil activation in fibrosing alveolitis: a comparison of lone cryptogenic fibrosing alveolitis and systemic sclerosis. Eur Respir J 1996; 9: 992-999.

29 Borregaard N, Cowland JB. Granules of the human neutrophilic polymorphonuclear leukocyte. Blood 1997; 89: 3503-3521.

30 Schmekel B, Karlsson SE, Linden M, et al. Myeloperoxidase in human lung lavage. I. A marker of local neutrophil activity. Inflammation 1990; 14: 447-454.

31 Kettritz R, Jennette JC, Falk RJ. Crosslinking of ANCA-antigens stimulates superoxide release by human neutrophils. J Am Soc Nephrol 1997; 8: 386-394.

32 Lynch JP III, Standiford TJ, Rolfe MW, et al. Neutrophilic alveolitis in idiopathic pulmonary fibrosis. The role of interleukin-8. Am Rev Respir Dis 1992; 145: 1433-1439.

33 Miller EJ, Cohen AB, Nagao S, et al. Elevated levels of NAP-1/ interleukin- 8 are present in the airspaces of patients with the adult respiratory distress syndrome and are associated with increased mortality. Am Rev Respir Dis 1992; 146: 427-432.

34 Ralston DR, Marsh CB, Lowe MP, et al. Antineutrophil cytoplasmic antibodies induce monocyte IL- 8 release. Role of surface 
proteinase-3, $\alpha_{1}$-antitrypsin, and Fc $\gamma$ receptors. J Clin Invest 1997; 100: 1416-1424.

35 Csernok E, Ernst M, Schmitt W, et al. Activated neutrophils express proteinase 3 on their plasma membrane in vitro and in vivo. Clin Exp Immunol 1994; 95: 244-250.

36 Zhou Z, Richard C, Menard HA. De novo synthesis of proteinase 3 by cytokine primed circulating human polymorphonuclear neutrophils and mononuclear cells. J Rheumatol 2000; 27: 2406-2411.

37 Loetscher P, Seitz M, Clark-Lewis I, et al. Both interleukin-8 receptors independently mediate chemotaxis. Jurkat cells transfected with
IL-8R1 or IL-8R2 migrate in response to IL-8, GRO- $\alpha$ and NAP-2. FEBS Lett 1994; 341: 187-192.

38 Hebert CA, Baker JB. Interleukin-8: a review. Cancer Invest 1993; 11: 743-750.

39 Liu L, Mul FP, Kuijpers TW, et al. Neutrophil transmigration across monolayers of endothelial cells and airway epithelial cells is regulated by different mechanisms. Ann NY Acad Sci 1996; 796: 21-29.

40 Pease JE, Sabroe I. The role of interleukin- 8 and its receptors in inflammatory lung disease: implications for therapy. Am J Respir Med 2002; 1: 19-25. 